\title{
Stability of Silicon carbide particle detector performance at elevated temperatures
}

\author{
Yusuf M. Abubakar, Student Member, IEEE, Annika Lohstroh, Member, IEEE, and Paul J. Sellin, Member, IEEE,
}

\begin{abstract}
The alpha spectroscopy performance and electric current stability of $\mathbf{4 H}$-silicon carbide Schottky devices with 50 $\mu \mathrm{m}$ epitaxial layer was examined at temperatures between 300 to $500 \mathrm{~K}$ at $50 \mathrm{~K}$ intervals. An activation energy of $5.98 \pm$ $0.64 \mathrm{meV}$ was extracted from temperature dependent resistivity measurements. The Schottky barrier height decreases from 1.33 $\mathrm{eV}$ at $300 \mathrm{~K}$ to $1.11 \mathrm{eV}$ at $500 \mathrm{~K}$ and the ideality factor increases from 1.17 at $300 \mathrm{k}$ to 1.79 at $500 \mathrm{~K}$. The reverse bias leakage currents stabilises faster at higher temperatures. The charge collection efficiency is above $90 \%$ for temperatures up to $500 \mathrm{~K}$. Pulse height spectra collected for 24 hours at constant voltage and temperature show improvements with time within the first 8 hours and remained stable for the remainder of the acquisition time. The peak width of the alpha spectra reduces significantly with increasing temperature at applied bias voltages below 50 $V$, which indicates that leakage currents are not the limiting factor in those conditions even at $500 \mathrm{~K}$ in our set up. So far, the devices indicate reasonable stability for extended periods of operation and highlight possible applications in harsh radiation media.
\end{abstract}

Index Terms - Silicon carbide, harsh radiation media, Schottky barrier height, ideality factor, epitaxial semiconductors, stability, noise.

\section{INTRODUCTION}

$\mathbf{S}$ ILICON carbide $(\mathrm{SiC})$ devices have been receiving considerable attention in recent years because of their properties that make them excellent candidates for harsh radiation media applications. Harsh radiation media are characterised by high temperature, high radiation fluence, high pressure and sometimes vibration. These conditions are present in nuclear reactor cores and oil and gas prospecting. Due to their wide band gap (3.2 eV for $4 \mathrm{H}-\mathrm{SiC}$ at room temperature) [1], limited leakage current even at elevated temperatures (in excess of $200^{\circ} \mathrm{C}$ ) [2], $\mathrm{SiC}$ devices are ideal for harsh environmental applications. In addition, the devices are chemically and mechanically stable [2]-[4]. They are widely used for nuclear radiation detection [5]-[11] . Other potential areas requiring High Temperature Electronics (HTE) include nuclear well logging, aerospace and space craft as well as automobile industries. The well logging sector is one of the largest areas that operates at high temperatures. The temperature ranges from $75^{\circ} \mathrm{C}$ up to $312^{\circ} \mathrm{C}$, for logging that takes longer periods [12], [13]. This increase in required operating temperature

Manuscript received August 25, 2015; revised Month? day?, 2015. This work was funded by the Petroleum Technology Development Fund-Nigeria.

Y.M./Abubakar is with the Department of Physics, Ahmadu Bello University Zaria and Department of Physics, member of the South East Physics Network (SEPnet), University of Surrey, Guildford, GU2 7XH, UK, e-mail: y.abubakar@surrey.ac.uk.

A. Lohstroh, and P. J./ Sellin are with the Department of Physics, member of the South East Physics Network (SEPnet), University of Surrey, Guildford, GU2 7XH, UK. is expected to continue to match the corresponding increase in the desired depth of investigation. In addition to the high temperature exposure, sometimes sensors are exposed to vibrations and shocks [14]. Therefore, demand for new materials and technology that can cope with harsh radiation environments is one of the central challenges in nuclear radiation detection applications.

The characteristics of the metal-semiconductor interface are as important as the charge transport properties of the bulk material because the devices are subjected to harsh environments. The two important parameters are Schottky barrier height (SBH) and ideality factor (IF). Ha and Kim [15] observed spatial non-uniformity in barrier height for $4 \mathrm{H}-$ $\mathrm{SiC} / \mathrm{Ni}$ interface with increasing temperature. They believed that the cause of this anomaly arises from potential fluctuations caused by crystal defects, inhomogeneous doping and other related defects. On the other hand, the model suggested by Tung [16] predicts the presence of non-uniform regions or patches locally, with relatively low and high barrier height. Since IF and SBH are strongly dependent on temperature, Gaussian distribution suggested by Werner and Güttler [17] can successfully explain the I-V(T) characteristics of semiconductor devices [18].

One of the earliest works on the suitability of using $\mathrm{SiC}$ for high temperature applications was conducted by Babcock and Chang [19]. The study involves exposing a $\mathrm{SiC}(\mathrm{p}-\mathrm{n}$ junction) detector to alpha radiation at high temperatures; extending up to $800{ }^{\circ} \mathrm{C}$. They observed that the magnitude of capacitance increases as the temperature is raised, implying that more carriers become ionized. Because they expected too much noise generation as a result of thermal agitation, they recorded the alpha spectra at high temperatures at zero bias voltage. This restriction of bias voltage would deny the chance of knowing the effect of temperature on the voltage dependent charge collection efficiency (CCE). In addition, a single cycle (heating and cooling once) was used for the high temperature measurement, limiting the understanding of structural changes in the devices as a result of heating and cooling. Garcia et al. [20] demonstrated the possibility of operating the devices for more than 10 cycles; however they suggested further investigation for extended periods of exposure, using devices with increased thickness of Schottky contacts (i.e. $200 \mathrm{~nm}$ ). In a similar development, Ha and Kim [21] investigated the thermal dependence of properties of p-n-n-type $\mathrm{SiC}$ detector for application in harsh environments. Their measurement of alpha spectra above room temperature was also carried out at zero bias. The reduction in CCE was relatively small but, there was significant broadening of the peak beyond 12 Volts bias. In an attempt to to use a UV SiC detector for radiation 
detection in harsh media, Metzger et al. [22] exposed 6H-SiC UV detector to Gamma-rays, protons and neutrons radiation under high temperature. The response (induced current versus dose rate) of the device was linear with respect to Gamma radiation at high temperatures up to $200{ }^{\circ} \mathrm{C}$. With $1000 \mathrm{kGy}$ (Air) dose of Gamma-rays, $8.5 \times 10^{12} \mathrm{~cm}^{-2}$ fluence of $32 \mathrm{MeV}$ protons and $4.1 \times 10^{12} \mathrm{~cm}^{-2}$ fluence of $14 \mathrm{MeV}$ neutrons, the device performance did not change significantly. Beyond 200 ${ }^{\circ} \mathrm{C}$, the response of the device was significantly affected.

Other studies conducted at high temperature involve the use of Schottky SiC devices operated at about $100{ }^{\circ} \mathrm{C}$. Ruddy et al. [23] compared the alpha detection properties of Schottky and p-n junction $(4 \mathrm{H}-\mathrm{SiC})$ devices between 22 and $89{ }^{\circ} \mathrm{C}$ and discovered that the Schottky devices have better resolution than the p-n junction ones. Additionally no temperature effect was noticed in the devices. This was supported by Nava et al. [24], who used 4H-SiC Schottky devices for alpha and beta detection at increased temperatures up to $94{ }^{\circ} \mathrm{C}$. In the most recent investigation, Kalinina et al. [25] demonstrated that an Al-ion implanted $\mathrm{p}-\mathrm{n}$ junction $(\mathrm{SiC})$ can be operated effectively in the temperature range of $20-500{ }^{\circ} \mathrm{C}$. They also established that CCE increases with raising temperature and the overall noise of the system reduces at higher temperatures. The former was attributed to an increase in holes diffusion length with temperature, while the latter was due to annealing of some radiation defects.

Although temperature effects are important in studying the performance of these devices, high radiation fluence (precisely protons and neutrons) has a greater effect on their detection ability. Nava et al. [26] have presented a comprehensive review in this regard. In the paper, particular emphasis was given to the CCE and resolution degradation of the devices under high radiation fluence.

In most of the studies published for charge particle radiation detection at high temperature using $\mathrm{SiC}$ devices, the observations are either limited to $100{ }^{\circ} \mathrm{C}$, or the spectral acquisition was carried out at single bias voltage. In some cases no voltage was applied at high temperature. Also, the long term stability of the devices at high temperatures has not been reported yet.

In this paper we report for the first time, high temperature stability studies of $4 \mathrm{H}-\mathrm{SiC}$ epitaxial detector used for alpha particles detection and the variation of CCE with applied bias under different temperatures. Charge transport properties as well as metal-semiconductor junction characteristics were also studied.

\section{MethoD}

The SiC Schottky device used in this research was processed by Alenia Marconi Systems (AMS) from $50 \mu \mathrm{m}$ thick n-type $4 \mathrm{H}-\mathrm{SiC}$ epitaxial layer $\left[N_{D}-N_{A}=5 \times 10^{14} \mathrm{~cm}^{-3}\right]$ grown on $\mathrm{n}^{+}$-type $4 \mathrm{HSiC}$ wafer (16-30 micropipes $/ \mathrm{cm}^{2}$ ) manufactured by Cree Inc. (see Fig. 1). Details of the fabrication processes are available in [27]. The diode of $10 \mathrm{~mm} \times 10 \mathrm{~mm}$ area was diced from a 2" substrate.

Current signal as a function of voltage applied to the top surface of the samples was measured in air at room temperature in the absence of light with a Keithley 487

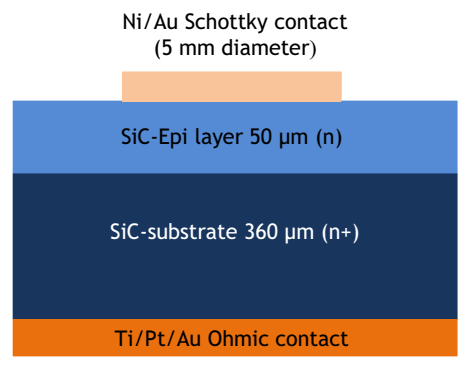

Fig. 1. Schematic representation of the $4 \mathrm{H}-\mathrm{SiC}$ device used in this study

picoammeter, integrated with a voltage source that was used as bias supply. The device under test (DUT) is normally connected in series to a $10 \mathrm{k} \Omega$ resistor (Figure 2). Additional leakage current was measured in vacuum from $300 \pm 0.1 \mathrm{~K}$ to $500 \pm 0.1 \mathrm{~K}$. The temperature was controlled by means of an Oxford Instruments' Microstat which comprises a cryostat, temperature controller connected to the hot finger in the cryostat and vacuum pump. Because of the voltage drop at the $10 \mathrm{k} \Omega$ resistor caused by relatively large leakage current in the forward bias, a voltage correction was made such that the voltage reaching the DUT is given by

$$
V=V_{a}-V_{d}
$$

where $V_{a}$ is the applied voltage and $V_{d}$ is the voltage drop across the series resistor. Also, the use of the $10 \mathrm{k} \Omega$ resistor slows down the breakdown effect by giving some time constant to the circuit. The ideality factor $n$, and barrier height $\Phi_{B}$,

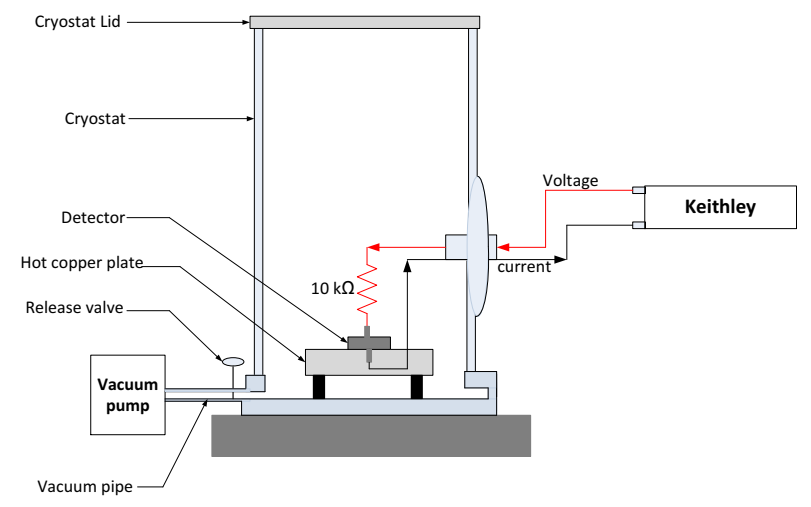

Fig. 2. Experimental set up for I/V measurement at elevated temperatures

were determined from thermionic emission theory equations 2 and $3[28]$

$$
\begin{gathered}
J=J_{s}\left(\exp \left(\frac{q V}{n k T}\right)-1\right) \\
J_{s}=A^{*} T^{2} \exp \left(-\frac{q \Phi_{B}}{k T}\right)
\end{gathered}
$$

where $A^{*}$ is the Richardson constant taken as $146 \mathrm{~cm}^{-2} \mathrm{~K}^{-2}$ for $\mathrm{SiC}$ [29], $V$ the forward voltage, $q$ electronic charge, $k$ 
Boltzmann's constant and $T$ the temperature. Current stability was measured by subjecting the devices to high temperatures for 20 minutes. Resistivity values extracted form the high temperature forward I/V curves were used to estimate the activation energy from equation 4 [30]

$$
\ln (\rho)=\ln \left(\rho_{0}\right)+\frac{E_{a}}{k T}
$$

where $E_{a}$ is the activation energy.

Capacitance of the devices was measured in air at room temperature without light interference by applying bias voltage to the top contact of the detector. An LCR meter (4284A) incorporated with bias supply was connected to the detector where it acts as voltage source while it measures the capacitance. Doping density $N_{D}$ and depletion thickness $w$ were obtained from equations 5 and 6 respectively [31]

$$
\begin{aligned}
N_{D} & =\frac{2}{q w A C \frac{d\left(1 / C^{2}\right)}{d V}} \\
w & =\frac{\epsilon_{0} \epsilon_{4 H-S i C} A}{C}
\end{aligned}
$$

where $A$ is the device area, $\epsilon_{0}$ permittivity of free space, $\epsilon_{4 H-S i C}$ material's relative permittivity (6.7 for $4 \mathrm{H}-\mathrm{SiC}$ [32]) and $C$ the capacitance.

An uncollimated radioisotope $\left({ }^{241} \mathrm{Am} \alpha\right.$-source) with an average emission energy of $3.76 \mathrm{MeV}$ and approximate activity of $60 \mathrm{kBq}$ was placed at about $20 \mathrm{~mm}$ above the $\mathrm{SiC}$ detector (see Fig. 3). This source suffers from energy degradation

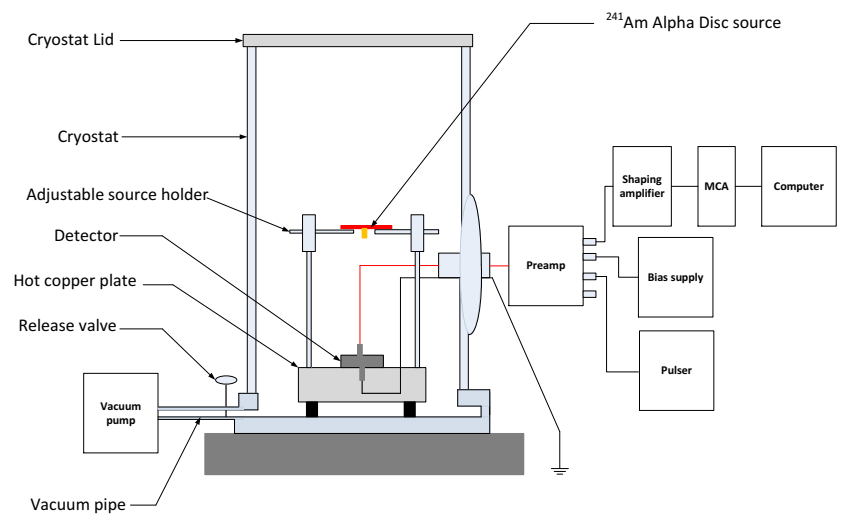

Fig. 3. Set-up used for the high temperature spectroscopy.

due to the nature of its encapsulation. The source is sealed within a disk of thin silver foil. This is in accordance with American National Standards Institute classification for high temperature rated sources. Spectral responses acquired from ${ }^{241} \mathrm{Am}$ alpha emitting sources using a Si-pin diode under the same condition including the one used in this study, have shown clearly the shift in the spectrum of the emission energy of the source in comparison to other sources. Fig. 4 is a comparison between the single alpha source used in this study $\left({ }^{241} \mathrm{Am}\right)$ and a triple alpha source $\left({ }^{239} \mathrm{Pu},{ }^{241} \mathrm{Am}\right.$ and $\left.{ }^{244} \mathrm{Cm}\right)$. The detector is biased via an Ortec $(142 \mathrm{~A})$

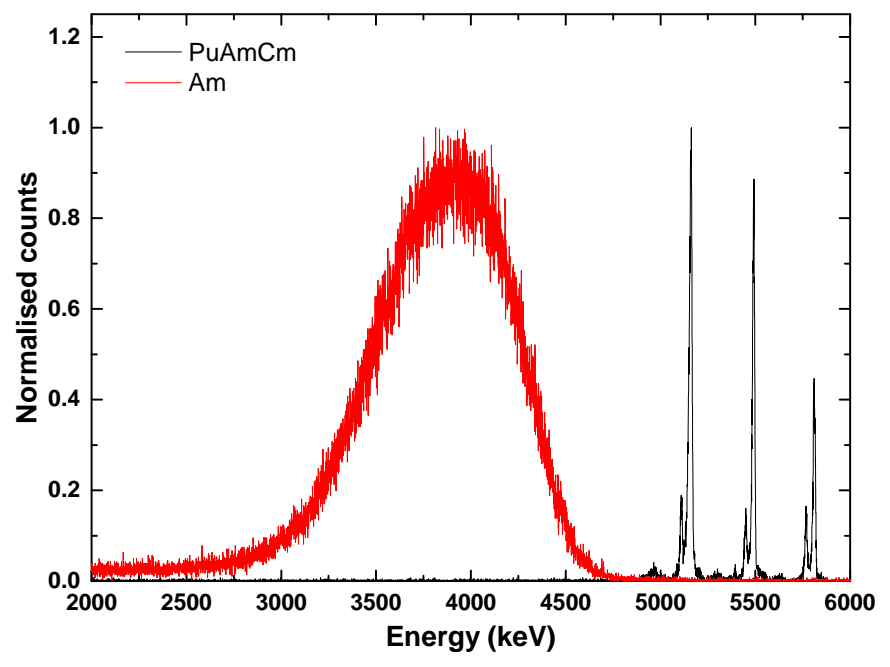

Fig. 4. Comparison of energy emission spectra between ${ }^{241} \mathrm{Am}$ and ${ }^{239} \mathrm{Pu}$, ${ }^{241} \mathrm{Am}$ and ${ }^{244} \mathrm{Cm}$ triple alpha radiation sources

charge sensitive preamplifier with its output signal connected (with a BNC cable) to the spectroscopy amplifier for signal shaping. Finally, the multi channel analyser converts the signal amplitude from the shaping amplifier into digital form which is displayed on a computer using MCA software. Alpha spectra were recorded for different applied bias voltages at different temperatures; ranging from 300 to $500 \mathrm{~K}$ at intervals of 50 $\mathrm{K}$. The radiation source and detector were placed inside a temperature controlled cryostat. In addition, ${ }^{241} \mathrm{Am}$ and pulser signal peaks were collected simultaneously by injecting signal form a 480 ORTEC pulser into the test input of the 142A ORTEC pre-amplifier. The broadening of the pulser peak recorded in this manner can be used to assess collectively the effect of detector leakage current and that of the noise form the front-end electronics to the overall resolution of the detector [33]. Further, stability of the signal was studied by fixing the values of temperature and bias voltage for longer periods up to 24 hours. Spectra were recorded at intervals of 20 minutes. In this work, we consider stability to be reached when the signal variations with time have dropped below $0.5 \%$ of the signal value.

\section{RESULTS AND Discussion}

\section{A. I/V and $C / V$ characteristics}

Current conduction in the devices shows clearly their Schottky behaviour at all temperatures. In the linear regime of the forward I/V characteristics, the gradient varies only between $5.40 \pm 0.42 \times 10^{-4}$ to $5.93 \pm 0.36 \times 10^{-4}(\Omega . c m)^{-1}$ over the whole temperature range (Fig. 5). The slow rising part of the forward I/V curve is as a result of barrier effect. In Fig. 6, bulk resistivity values are plotted against temperature, yielding an activation energy of $5.98 \pm 0.64 \mathrm{meV}$. The IF shows a consistent and linear increase with temperature up to $500 \mathrm{~K}$. Within this range the maximum and minimum values of IF are 1.17 and 1.79 respectively (Fig. 7). On the other hand, the SBH reduces with temperature increase from 1.33 to $1.11 \mathrm{eV}$ 

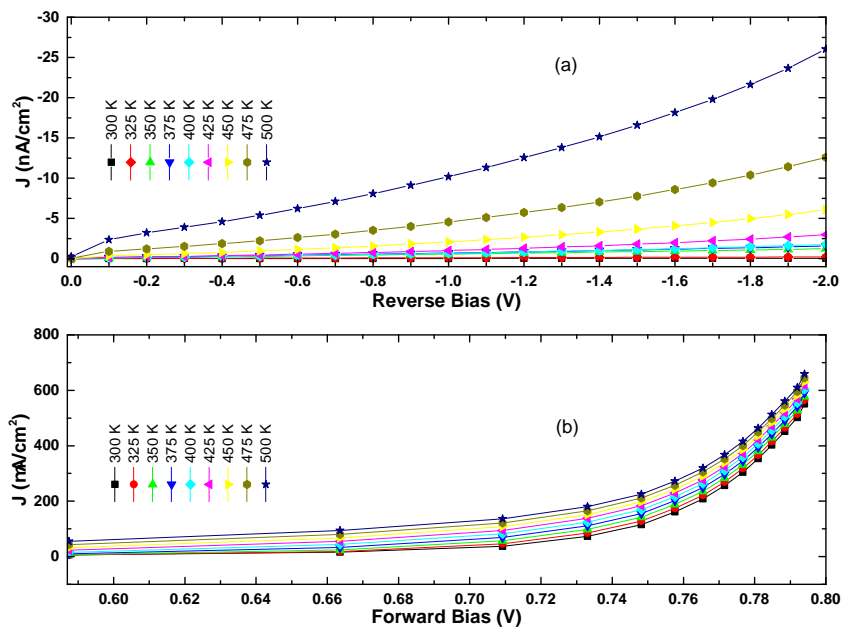

Fig. 5. (a) Forward current density at low voltages from 300-500 K; (b) Reverse current density measured at the same condition.

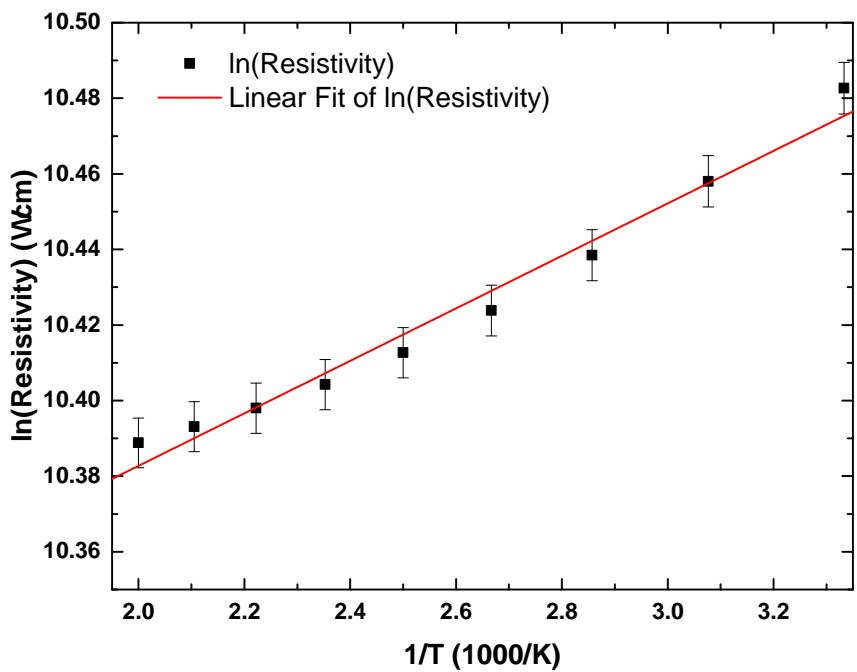

Fig. 6. Arrhenius plot with the fitted line that was used to calculate activation energy.

within the range of $300 \mathrm{~K}$ to $500 \mathrm{~K}$ (Fig. 7). The increase in ideality factor with increasing temperature indicates that thermionic emission is dominant and, similar result has been reported by Lee et al. [34]. As for the reduction in SBH, it is possible that the temperature increase has increased the effective dopant density in the device, which can reduce the SBH so that thermionic field emission could take place [35]. Generally, recombination current (under low forward biases) plays an important role in determining the departure of form ideal behaviour of schottky diodes [35].

Leakage current as a function of bias voltage at room temperature and above are presented in Fig. 8. The highest leakage current at room temperature is approximately $3 \mathrm{nA}$; when compared with the values obtained at higher temperatures, it can be seen that the leakage current rises up to about $200 \mathrm{nA}$. Leakage current (monitored for 20 minutes) reaches stable values faster at higher temperatures. As illustrated in

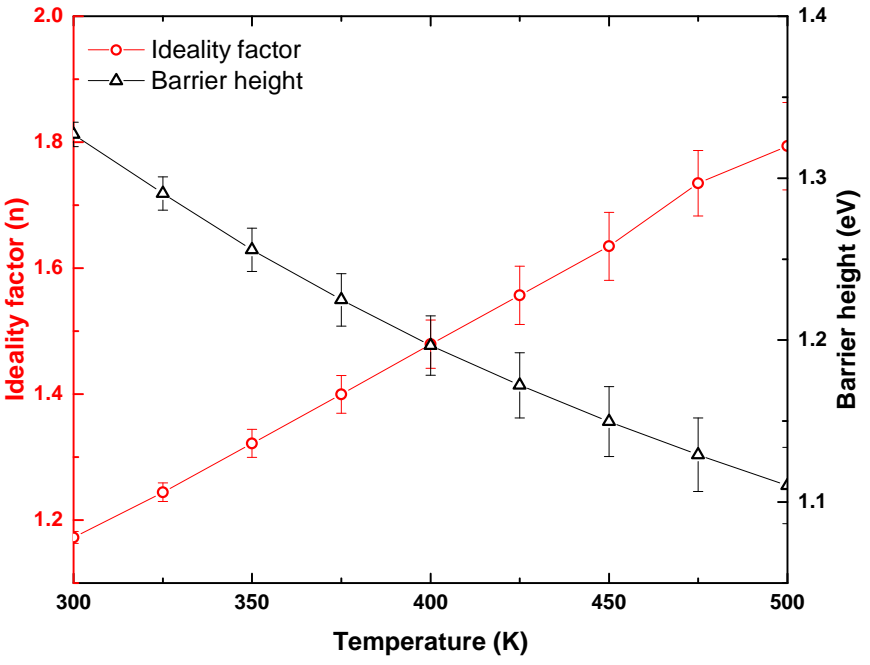

Fig. 7. Ideality factor and barrier height at different temperatures. Significant difference in the curves can be observed just after $400 \mathrm{~K}$.
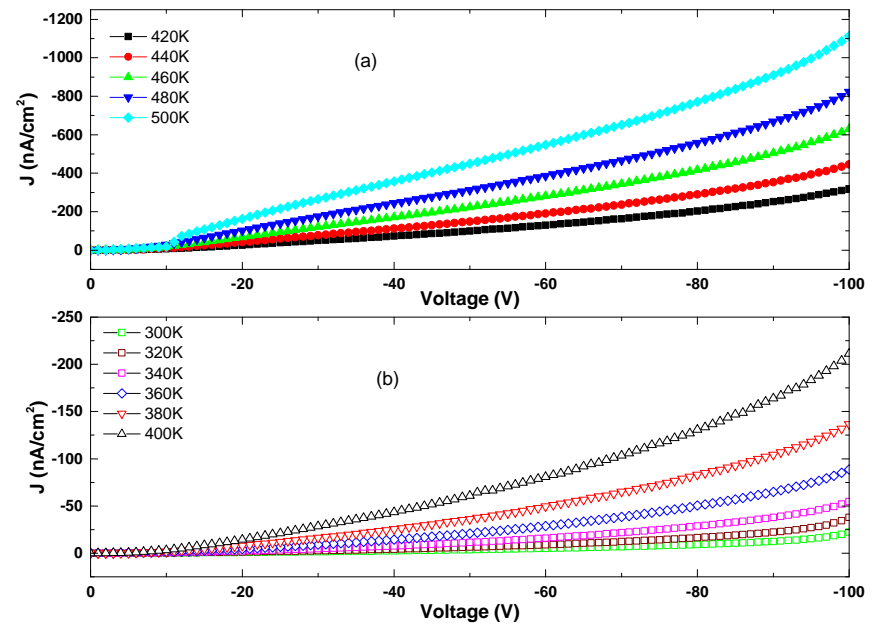

Fig. 8. Leakage current density as a function of reverse bias Voltage measured at elevated temperatures (a) from 300-400 K and (b) from 420-500K.

Fig. 9, it can be observed that at $500 \mathrm{~K}$ the current stabilizes just after the first two minutes while at lower temperatures it takes up to 8 minutes or more before stability is achieved. Disregarding the magnitude of the leakage current, it is clear that the operation of this device is favoured more at high temperatures.

The $1 / C^{2}$ versus voltage is linear, indicating that the doping concentration is constant throughout the active volume of the device yielding a value of $(16.3 \pm 0.8) \times 10^{14} \mathrm{~cm}^{-3}($ Fig. 10). The depletion thickness reaches $32.5 \mu \mathrm{m}$ at $40 \mathrm{~V}$. SRIM [36] simulation performed shows that the alpha particles with energy similar to the source used have an average range of $10.56 \mu \mathrm{m}$ (i.e., for $3.76 \mathrm{MeV}$ alpha particles). Therefore, from the measured and simulated results, bearing in mind the limitation of the measurement set up, there is full assurance that most of the alpha particles striking the device surface will cause ionization in the device's active volume. 

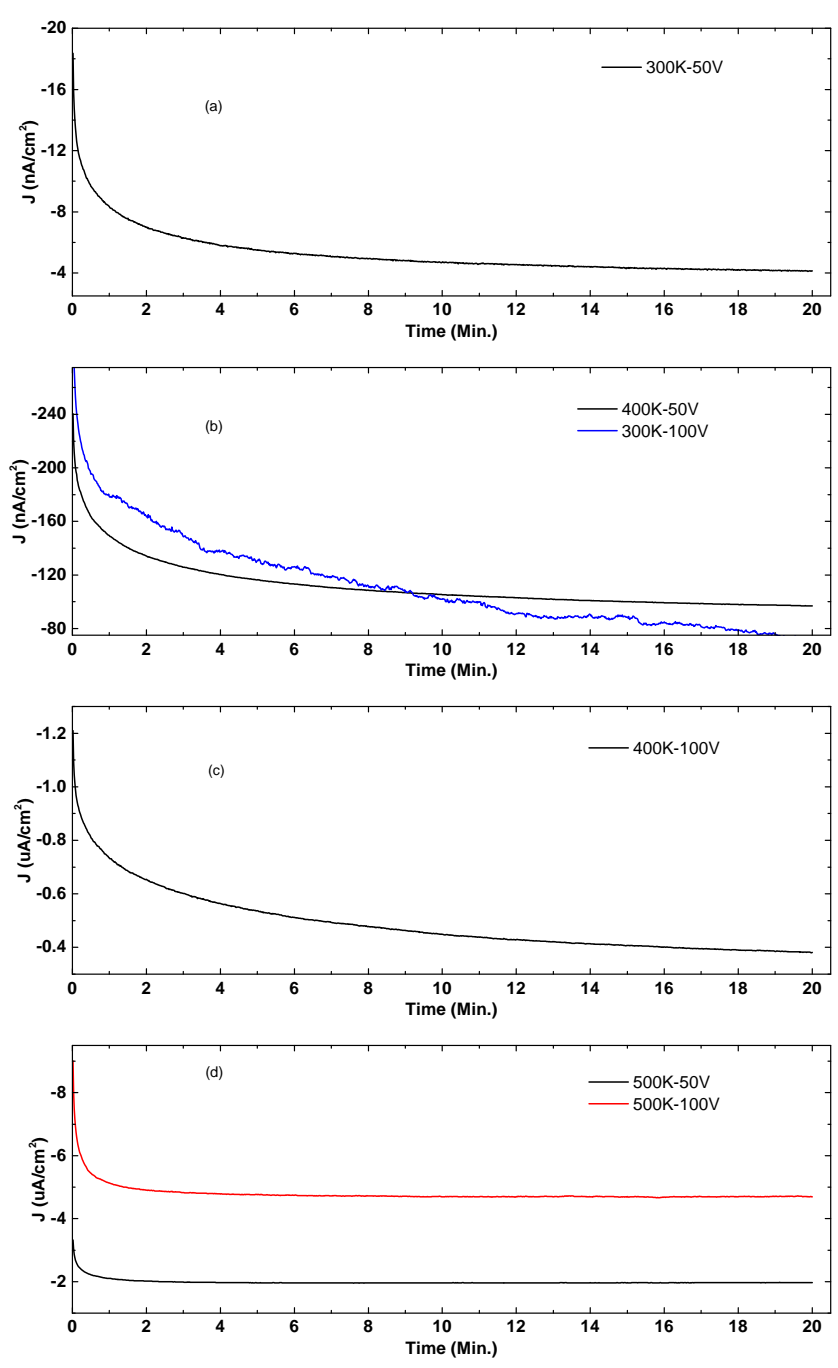

Fig. 9. Leakage current stability studies for 20 minutes at different bias voltages and temperatures. (a) $300 \mathrm{~K}, 50 \mathrm{~V}$ (b) $400 \mathrm{~K}, 50 \mathrm{~V}$ and $300 \mathrm{~K}, 100$ $\mathrm{V}$ (c) $400 \mathrm{k}, 100 \mathrm{~V}$ (d) $500 \mathrm{~K}, 50 \mathrm{~V}$ and $500 \mathrm{~K}, 100 \mathrm{~V}$.

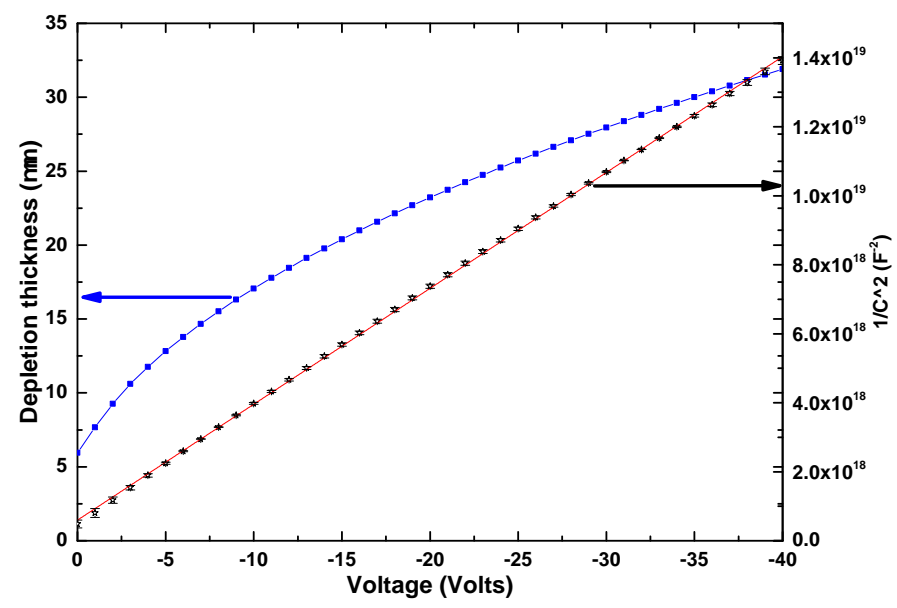

Fig. 10. Capacitance and depletion thickness as a function of applied bias measured at room temperature.

\section{B. Alpha spectroscopy}

In order to examine the contribution of leakage current to the overall noise in the system, pulser and ${ }^{241} \mathrm{Am}$ signals were recorded simultaneously and eventually full width at half maximum (FWHM) was extracted from the pulser peaks at various applied voltages and temperatures. The spectra acquired from both sources simultaneously are presented in Fig. 11. As observed from the figure, the pulser spectra
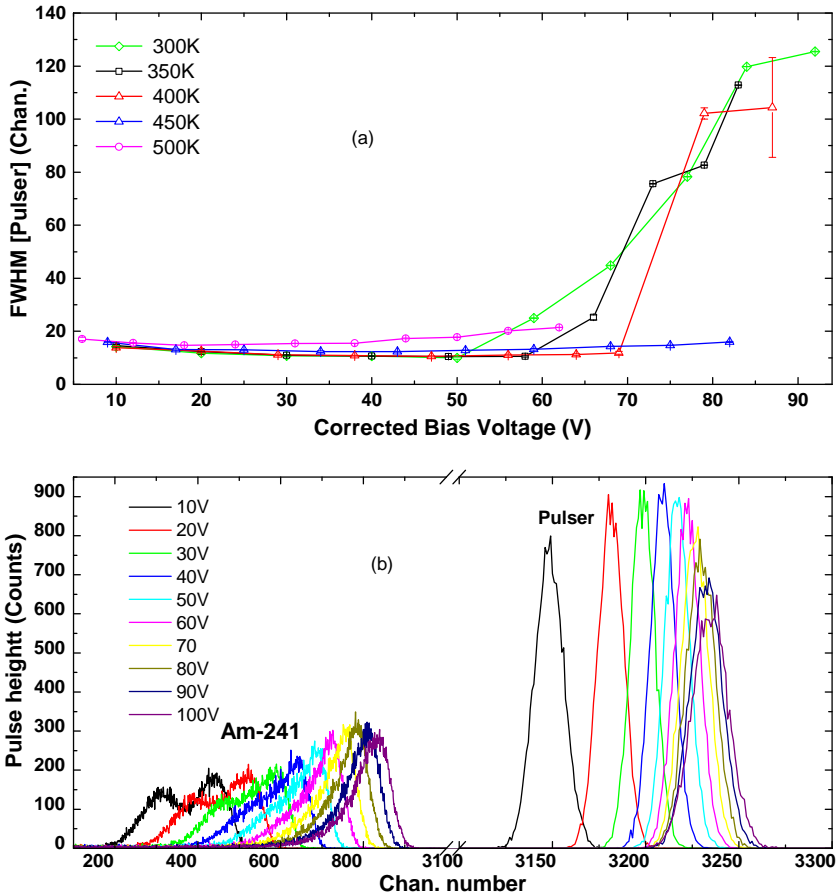

Fig. 11. (a) FWHM from pulser as a function of voltage at various temperatures; (b) pulser and alpha spectra recorded simultaneously at 500 $\mathrm{K}$.

acquired during the alpha irradiation indicate low FWHM fluctuation at higher operational temperatures. The FWHM from the pulser tends to be constant and significantly lower at 450 and $500 \mathrm{~K}$ than the room temperature value, even at $100 \mathrm{~V}$ bias. In contrast, FWHM increases with large values (more than 3 times initial value) beyond $50 \mathrm{~V}$ from 300 to approximately $400 \mathrm{~K}$. Nevertheless, at $50 \mathrm{~V}$ bias and below, the observed pulser signal width is minimum for all temperatures. The most important part of this result is the decrease in FWHM with increasing temperature affirming that there is limited contribution of leakage current to the overall energy resolution of the system. This is consistent with observations of Kalinina et al. [25] when they examined an aluminiumimplanted $\mathrm{p}-\mathrm{n}$ junction $\mathrm{SiC}$ detector, as mentioned earlier in section I. The CCE of the devices approaches $90 \%$ for most of the temperatures (see Fig. 12). Although the leakage current in the device at $500 \mathrm{~K}$ is low (less than $1 \mu \mathrm{A}$ ), the large input resistance of the pre-amplifier (100 M $\Omega$ ) [37] has caused significant voltage drop. This voltage drop was corrected using equation 1. Surprisingly, there is remarkable reduction in FWHM at higher temperatures. Comparing Fig. 11 to Fig. 12, it is evident that at $450 \mathrm{~K}$ and $500 \mathrm{~K}$, there is less effect of 


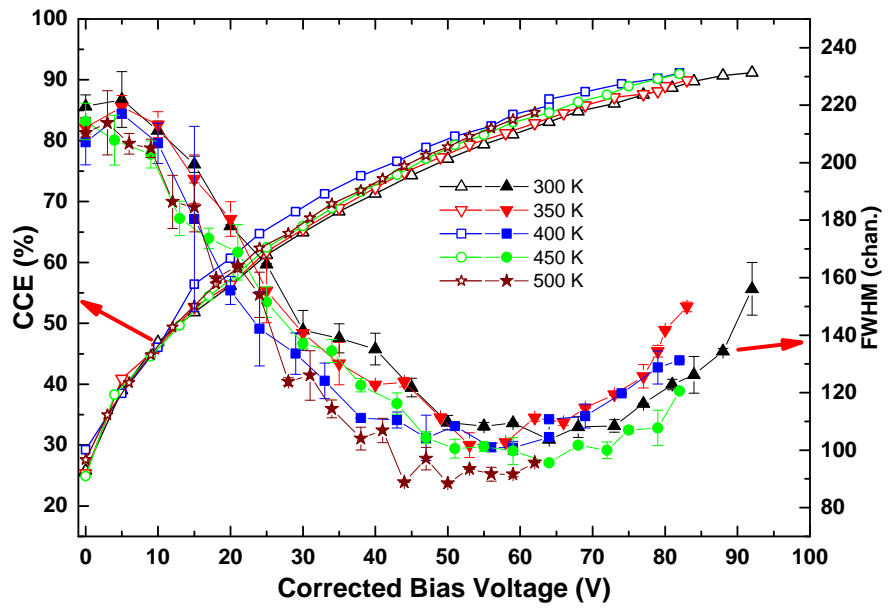

Fig. 12. CCE and FWHM for different bias voltages at different temperatures.

electronic noise to the FWHM at $90 \mathrm{~V}$.

Stability: At $500 \mathrm{~K}$ and $100 \mathrm{~V}$ applied bias, the normalised peak centroid position of ${ }^{241} \mathrm{Am}$ increases from 0.9 to 1.0 over 8 hours and remains stable afterwards (Fig. 13). Furthermore, the normalised pulse height variations after the first 8 hours is insignificant (with standard deviation of 0.0024) and a similar trend is observable in the normalised FWHM. At $450 \mathrm{~K}$ and $100 \mathrm{~V}$ bias the normalised peak centroid position of ${ }^{241} \mathrm{Am}$ reduces form 1 to 0.9 over 10 hours and remains stable afterwards. The difference in the time to reach stability for $450 \mathrm{~K}$ and $500 \mathrm{~K}$ may be as a result of charge trapping and de-trapping which could have reached an equilibrium at $500 \mathrm{~K}$. On the other hand, the device is barely stable at temperatures

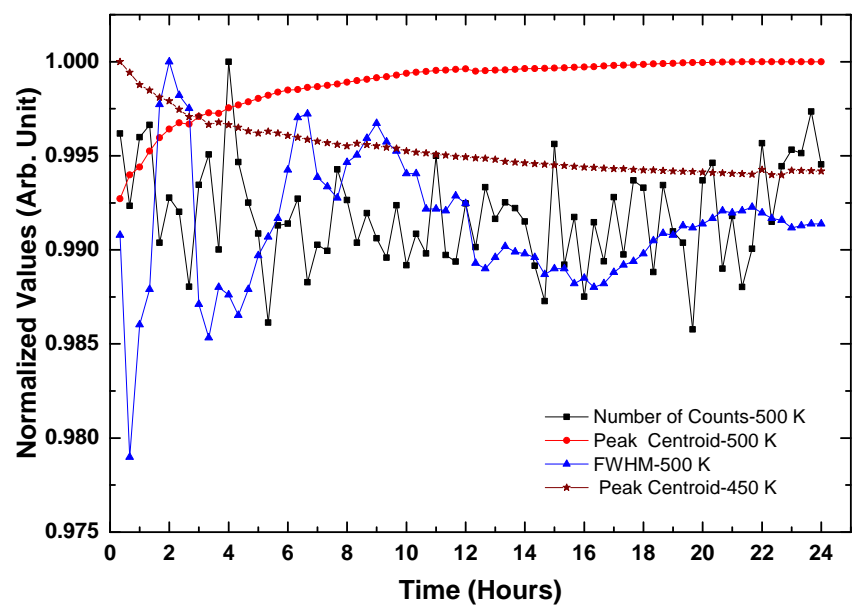

Fig. 13. Signal stability monitored for 24 hours at $500 \mathrm{~K}$ and $100 \mathrm{~V}$ bias. All the parameters were normalized to their highest value. The maximum values at $500 \mathrm{~K}$ of counts was $1.545 \times 10^{5}$, the peak centroid position $833.5 \mathrm{ch}$ and the FWHM $101.5 \mathrm{ch}$ while the maximum peak centroid position at 450 $\mathrm{K}$ and $100 \mathrm{~V}$ bias is $875.1 \mathrm{ch}$. The voltage applied here is uncorrected one. The levelling off of the centroid can be observed after 6 hours

below $450 \mathrm{~K}$ even at $50 \mathrm{~V}$ bias. Therefore, it is evident from this result that the device becomes stable with increasing temperature.

Looking at the stability of the alpha signal, leakage current and the overall energy resolution from the system, it can be established that the devices are more stable under high temperatures. Thus, it may be possible to optimize the devices for harsh radiation applications, precisely where there is need for longer operation time.

\section{CONCLUSION}

This work reports an initial study on the stability of alpha particle induced signal over a 24 hour period at temperatures up to $500 \mathrm{~K}$ at non-zero bias, which is relevant for applications of $\mathrm{SiC}$ diodes for oil and gas prospecting and nuclear reactor monitoring. A high quality epitaxial Schottky $\mathrm{SiC}$ device has been characterized for particle detection at high temperature and extended periods. The I/V and $\mathrm{C} / \mathrm{V}$ characteristics imply a normal behaviour expected from this type of Schottky diode. $\mathrm{BH}$ decreases with increasing temperature while IF increases with increasing temperature. There was a small variation in resistivity of the device with temperature increase yielding an activation energy of $5.98 \pm 0.64 \mathrm{meV}$. Approximately $90 \%$ CCE was achieved for all measurement temperatures. The contribution of leakage current to the energy resolution in the system becomes relatively low at high temperature and low bias voltages (i.e. $\leq 50 \mathrm{~V}$ ); whereas at $450 \mathrm{~K}$ and $500 \mathrm{~K}$ the FWHM was almost constant up to approximately $80 \mathrm{~V}$. The alpha spectra recorded for 24 hours vary with time for the first eight hours and, remain stable for the remaining 18 hours. Similarly, the leakage current stability was reached faster at higher temperatures. Hence, high temperature operation of the studied SiC diode operated as charge particle detectors resulted in better stability and energy resolution than at room temperature. This further confirms the potential of SiC diodes for oil and gas prospecting and future work will need to show whether this result can be extended to other types of $\mathrm{SiC}$ diodes and whether the performance can be maintained under vibration.

\section{ACKNOWLEDGMENT}

The authors would like to thank Stefania Miglio and Mara Bruzzi for supplying the samples. The technical support provided by John-William Brown, Robert Derham, Bob Norton, Steve Craig and Simon Barnes is well appreciated.

\section{REFERENCES}

[1] P. G. Neudeck, "Progress towards high temperature, high power SiC devices," in Compound Semiconductors 1994, Proceedings of the TwentyFirst INT Symposium on Compound Semiconductors held in San Diego, California, 18-22 September 1994, vol. 18. CRC Press, 1995, p. 22.

[2] R. Chand, M. Esashi, and S. Tanaka, "PN junction and metal contact reliability of SiC diode in high temperature $(873 \mathrm{~K})$ environment," SolidState Electronics, vol. 94, no. 0, pp. 82 - 85, 2014. [Online]. Available: http://www.sciencedirect.com/science/article/pii/S0038110114000215

[3] M. Mehregany, C. Zorman, N. Rajan, and C. H. Wu, "Silicon carbide MEMS for harsh environments," Proceedings of the IEEE, vol. 86, no. 8, pp. 1594-1609, 1998. [Online]. Available: http: //ieeexplore.ieee.org/xpl/articleDetails.jsp?arnumber=704265

[4] Y. T. Yang, K. L. Ekinci, X. M. H. Huang, L. M. Schiavone, M. L. Roukes, C. A. Zorman, and M. Mehregany, "Monocrystalline silicon carbide nanoelectromechanical systems," Applied Physics Letters, vol. 78, no. 2, pp. 162-164, 2001. [Online]. Available: http://scitation.aip.org/content/aip/journal/apl/78/2/10.1063/1.1338959 
[5] M. Bruzzi, F. Nava, S. Russo, S. Sciortino, and P. Vanni, "Characterisation of silicon carbide detectors response to electron and photon irradiation," Diamond and Related Materials, vol. 10, no. 37, pp. 657661, 2001.

[6] M. Bruzzi, S. Lagomarsino, F. Nava, and S. Sciortino, "Characterisation of epitaxial SiC Schottky barriers as particle detectors," Diamond and Related Materials, vol. 12, no. 3-7, pp. 1205-1208, 2003.

[7] W. Cunningham, J. Melone, M. Horn, V. Kazukauskas, P. Roy, F. Doherty, M. Glaser, J. Vaitkus, and M. Rahman, "Performance of irradiated bulk SiC detectors," Nuclear Instruments \& Methods in Physics Research Section A: Accelerators Spectrometers Detectors and Associated Equipment, vol. 509, no. 1-3, pp. 127-131, 2003.

[8] R. Flammang, J. Seidel, and F. Ruddy, "Fast neutron detection with silicon carbide semiconductor radiation detectors," Nuclear Instruments and Methods in Physics Research Section A: Accelerators, Spectrometers, Detectors and Associated Equipment, vol. 579, no. 1, pp. 177-179, 2007.

[9] P. G. Mcmullin, D. L. Barrett, R. H. Hopkins, J. A. Spitznagel, J. A. Powell, and F. V. Thome, "Silicon-Carbide Devices for Radiation Hard Applications," 10th Symposium on Space Nuclear Power and Propulsion, Pts 1-3, vol. 271, pp. 625-630, 1993.

[10] F. Moscatelli, "Silicon carbide for UV, alpha, beta and X-ray detectors: Results and perspectives," Nuclear Instruments and Methods in Physics Research Section A: Accelerators, Spectrometers, Detectors and Associated Equipment, vol. 583, no. 1, pp. 157-161, 2007.

[11] M. Rogalla, K. Runge, and A. Sldner-Rembold, "Particle detectors based on semi-insulating silicon carbide," Nuclear Physics B-Proceedings Supplements, vol. 78, no. 1, pp. 516-520, 1999.

[12] N. R. Z. Abidin, E. D. C. K. Yew, and Z. Lockman, "Material selection for High temperature electronic devices and Its potential applications," Feature High Temperature, pp. 6-12, 2009.

[13] A. Nikitin and S. Bliven, "Needs of well logging industry in new nuclear detectors," 2010 IEEE Nuclear Science Symposium Conference Record (Nss/Mic), pp. 1214-1219, 2010.

[14] F. Touati, F. Mnif, and A. Lawati, "High-Temperature Electronics: Status and Future Prospects in the 21st Century," The Journal of Engineering Research, vol. 3, no. 1, pp. 43-54, 2006. [Online]. Available: http://icccp05.tjer.net/issue3/p6.pdf

[15] J. H. Ha and H. S. Kim, "Schottky Barrier Inhomogeneities of a $4 \mathrm{~h}-\mathrm{SiC} / \mathrm{Ni}$ Contact in a Surface Barrier Detector," Journal of the Korean Physical Society, vol. 58, no. 2, p. 205, 2011. [Online]. Available: http://www.kps.or.kr/jkps/abstract_view.asp? articleuid=6ACA058A-72FC-47AD-9F67-4831B255A991

[16] R. Tung, "Electron transport at metal-semiconductor interfaces: General theory," Physical Review B, vol. 45, no. 23, p. 13509, 1992.

[17] J. H. Werner and H. H. Güttler, "Barrier inhomogeneities at Schottky contacts," Journal of applied physics, vol. 69, no. 3, pp. 1522-1533, 1991.

[18] S. Khannaa, S. Neeleshwara, and A. Noor, "Current Voltage-Temperature (IVT) characteristics of $\mathrm{Cr} / 4 \mathrm{~h}-\mathrm{SiC}$ Schottky diodes," Journal of Electron Devices, vol. 9, pp. 382-389, 2011.

[19] R. Babcock and H. Chang, "SiC neutron detectors for high-temperature operation," Neutron Dosimetry, vol. 1, no. 1, p. 613, 1963.

[20] T. R. Garcia, A. Kumar, B. Reinke, T. E. Blue, and W. Windl, "Electronhole pair generation in SiC high-temperature alpha particle detectors," Applied Physics Letters, vol. 103, no. 15, p. 152108, 2013.

[21] J. H. Ha and H. S. Kim, "Alpha-ray spectrometry at high temperature by using a compound semiconductor detector," Applied Radiation and Isotopes, vol. 81, pp. 165-168, 2013.

[22] S. Metzger, H. Henschel, O. Kohn, and W. Lennartz, "Silicon carbide radiation detector for harsh environments," IEEE Transactions on Nuclear Science, vol. 49, no. 3, pp. 1351-1355, 2002.

[23] F. Ruddy, A. Dulloo, J. Seidel, S. Seshadri, and L. Rowland, "Development of a silicon carbide radiation detector," IEEE Transactions on Nuclear Science, , vol. 45, no. 3, pp. 536-541, 1998

[24] F. Nava, P. Vanni, M. Bruzzi, S. Lagomarsino, S. Sciortino, G. Wagner, and C. Lanzieri, "Minimum ionizing and alpha particles detectors based on epitaxial semiconductor silicon carbide," IEEE Transactions on Nuclear Science, vol. 51, no. 1, pp. 238-244, 2004.

[25] E. Kalinina, A. Ivanov, N. Strokan, and A. Lebedev, "Structure and characteristics of the high-temperature $\mathrm{SiC}$ detectors based on $\mathrm{Al}$ ion-implanted $\mathrm{p}+\mathrm{n}$ junctions," Semiconductor Science and Technology, vol. 26, no. 4, p. 045001, 2011.

[26] F. Nava, G. Bertuccio, A. Cavallini, and E. Vittone, "Silicon carbide and its use as a radiation detector material," Measurement Science and Technology, vol. 19, no. 10, p. 102001, 2008.
[Online]. Available: http://stacks.iop.org/0957-0233/19/i=10/a=102001? key $=$ crossref $.8731 \mathrm{f} 15 \mathrm{c} 5 \mathrm{cdec} 6 \mathrm{ff} 858488 \mathrm{ab} 42255 \mathrm{cef}$

[27] A. L. Giudice, "Fabrication and characterization of wide area SiC detectors for neutron monitoring," University of Turin, Italy, CERN, 4th RD50 Workshop, 2004. [Online]. Available: http: //rd50.web.cern.ch/rd50/4th-workshop/talks/7-3-RD50_LoGiudice.pdf

[28] S. M. Sze, Semiconductor devices: physics and technology, 3rd ed. Singapore: John Wiley \& Sons, 2013.

[29] A. Itoh, T. Kimoto, and H. Matsunami, "High performance of highvoltage 4h-SiC Schottky barrier diodes," Electron Device Letters, IEEE, vol. 16, no. 6, pp. 280-282, 1995.

[30] D. Roy, S. Das, and P. Nandy, "Possibility of decreasing the activation energy of resistivity of mullite by doping with nickel ion," Materials Science-Poland, vol. 30, no. 4, pp. 406-413, 2012.

[31] G. Lutz, Seimconductor Radiation Detectors: Device Physics. Springer, 1999.

[32] O. Madelung, Semiconductors: data handbook, 3rd ed. Germany: Springer Science \& Business Media, 2004

[33] S. K. Chaudhuri, K. J. Zavalla, and K. C. Mandal, "Experimental determination of electron-hole pair creation energy in $4 \mathrm{~h}-\mathrm{SiC}$ epitaxial layer: An absolute calibration approach," Applied Physics Letters, vol. 102, no. 3, p. 031109, 2013.

[34] S.-K. Lee, C.-M. Zetterling, and M. Östling, "Schottky diode formation and characterization of titanium tungsten to n- and p-type $4 \mathrm{~h}$ silicon carbide," Journal of Applied Physics, vol. 87, no. 11, pp. 8039-8044, 2000. [Online]. Available: http://scitation.aip.org/content/aip/journal/jap/ 87/11/10.1063/1.373494

[35] E. H. Rhoderick and R. Williams, Metal-semiconductor contacts. Clarendon Press Oxford, 1988.

[36] J. F. Ziegler, M. Ziegler, and J. Biersack, "SRIM The stopping and range of ions in matter," Nuclear Instruments and Methods in Physics Research Section B: Beam Interactions with Materials and Atoms, vol. 268, no. 11, pp. 1818-1823, 2010.

[37] Advanced Measurement Technology, Inc., "Models 142a, 142b, and 142c Preamplifiers Operating and Service Manual," 2002. [Online]. Available: http://www.qsl.net/k/k0ff/01\%20Manuals/NIM\%20Manuals/ NIM/ORTEC/Ortec-142ABC-MNL.pdf 